\title{
En busca de los contenidos de la Educación Física
}

Este artículo presenta una serie de apuntes sobre las temáticas que orientaron el saber específico de la disciplina en la Facultad de Educación Física de la Universidad Pedagógica Nacional, y que pueden haber ayudado a configurar un objeto de estudio amplio, como base del proyecto curricular aprobado en 1984. La descripción se basa en las referencias teóricas relacionadas con cada contenido significativo de cada momento, y en las reacciones internas en la Facultad, que pueden haber determinado orientaciones de formación de los profesionales en educación física, egresados en los últimos 25 años.

Palabras clave: Educación física, currículo, saber, tendencia, gimnasia, danza, condición física, deporte, psícomotricidad, fisiología del ejercicio, teoría de la educación física, teoría del entrenamiento, ciencia del deporte, corporeidad.

La formación de Profesores de Educación física ha sido tarea central de la actual Facultad de Educación Física (FEF) de la Universidad Pedagógica Nacional durante más de 50 años. Esta misión ha sido desarrollada a través de distintos currículos de formación, los cuales fueron concebidos en diferentes épocas y desde concepciones diversas. Sin embargo, a pesar de los distintos enfoques de cada momento, es posible que la disciplina haya ido encontrando contenidos significativos para su proceso de construcción, que representan núcleos temáticos que ayudaron a resolver problemas del saber específico de la EF (educación física) a lo largo de la vida de la Facultad.

Se podrían plantear ciertos temas que fueron contenidos del desarrollo académico en EF, durante la época de referencia (1976-2000): la gimnasia, las danzas, la condición física, los deportes, la psicomotricidad, la fisiología del ejercicio, la teoría de la educación física, la teoría del entrenamiento, las ciencias del deporte (sociología del deporte, antropología deportiva, psicología deportiva, biomecánica deportiva, fisiología del deporte), y la corporeidad.

Aunque se han destacado los anteriores, algunos de ellos engloban otros. Es decir, estos campos de estudio pueden contener subcampos que les son propios y que se reconocen como tales en el ámbito de la EE Por ejemplo, en la Teoría de la Educación física, el campo de los estudios sobre el movimiento. 
La gimnasia fue el primer tema que determinó la orientación curricular en EF Es claro que su desarrollo en Europa fue determinante en la conformación de las bases de la EF suramericana y en particular de la colombiana. En efecto, los primeros profesionales de la Escuela Nacional de Educación Física y luego del Departamento de EF (origen de la actual Facultad) fueron formados con una gran base académica en gimnasia.

El currículo presentaba en los años 70 una gran cantidad de asignaturas en gimnasia: Gimnasia I,

Gimnasia II, Gimnasia Olímpica I, Gimnasia Olímpica II, Gimnasia Rítmica, Gimnasia Infantil y

Gimnasia estética. Es en esta época que los fundamentos biológicos de la EF se instalan en la

formación de los profesores de EF El núcleo de la Gimnasia se mantuvo en el plan de 1984, en

cierta manera como símbolo del origen de la educación física moderna, y como actividad real de

la práctica de la educación física.

Las danzas. Ha sido un tema constantemente presente en el programa de formación. Su aparición en el currículo de antes del 84 y su orientación se debe a la Profesora Ana María Chávez Posada, quien distinguió las Danzas en Nacionales y Extranjeras. Su enfoque correspondió al del desarrollo de la cultura folclórica. El plan de 1984 acogió el tema como parte de la EE reconociendo que se podía estudiar la Teoría y Método de las Danzas y luego profundizar en tendencias distintas en un nivel li de Danzas. No hay que olvidar que luego del apogeo de la Gimnasia en Europa, una corriente o movimiento propuso y desarrolló las Danzas como alternativa de formación pedagógica en EF.

La condición física fue un tema que trascendió en Norteamérica, bajo la idea de la "buena vida» (en el sentido de una calidad de vida elevada). Este concepto incluía los aspectos físicos como salud positiva (fitness) y la habilidad para moverse eficientemente en trabajo, juego y locomoción (performance). Se desarrolló a través de investigaciones, surgidas del problema de la falta de condición de los jóvenes norteamericanos, evidenciada en el test de condición muscular mínima entre 1950 y 1960 . Ya era una preocupación desde 1954, cuando Krauss y Hirschland estudiaron el tema y encontraron en inferiores condiciones a los jóvenes norteamericanos comparados con los europeos de la época ${ }^{1}$. Estas investigaciones, se calcula, estudiaron más de 20 millones de escolares por año en los finales de los 70.

La relación entre salud y actividad física es un tema que circuló internacionalmente y de alguna

manera influyó en Colombia y en la UPN. Se trataba de una tendencia con base en investigación

de corte empírica analítica. Es posible que el enfoque se conservara en el modo de seleccionar y

evaluar en EF El concepto de valencias físicas y el complejo VARFEC (velocidad, agilidad,

\footnotetext{
${ }^{1}$ Anotaciones sobre el "Manual del test de la AAHPERD". 
resistencia, fuerza, equilibrio y coordinación) determinaron que los licenciados de la época

consideraran éste aspecto como eje de su trabajo profesional.

El control motor y el aprendizaje motor es otro tema de estudio en los 80. La preocupación sobre el movimiento, como un aspecto crítico de la vida, originó una serie de investigaciones sobre cómo los movimientos son «controlados», como la información sensorial del ambiente y/o del cuerpo es utilizada en tal control, y cómo tal información permite a una persona seleccionar un movimiento ${ }^{2}$

Al añadir posteriormente el estudio de cómo los movimientos son aprendidos, surge el tema del aprendizaje motor. Esta tendencia norteamericana se sitúa en el campo de la Psicología Conductista. Incluso Singer $^{3}$, continuó esta línea, que además permanece durante los 80 en Norteamérica.

La corriente europea sobre el tema se desarrolla como psicomotricidad, desde bastiones como las escuelas de medicina (servicios de neurología) ${ }^{4}$ y des de aplicaciones como la reeducación motriz o el retraso mental ${ }^{5}$. La versión adelantada de esta corriente investigativa es la de LeBoulch, quien propone la relación entre desarrollo mental y movimiento, llegando a un planteamiento sobre el papel expresivo del movimiento. ${ }^{6}$ En Canadá se propone una versión sobre la motricidad a finales de los 80 , desde una postura más fisiológica pero que incorpora temas como la lateralidad, el desarrollo motor, la escritura y la organización espacio-temporal ${ }^{7}$.

Lo cierto es que la psicomotricidad se instaló como asignatura específica y obligatoria de la li-

cenciatura, y como orientación preferencial de la práctica docente en el Departamento de la

época. No se concebía un profesional de la EF que no planeara y realizara su trabajo con los

elementos conceptuales de la psicomotricidad: esquema corporal, ajuste postural, conductas

motrices de base (coordinación y equilibrio), conductas perceptivo motrices (construcción de las

nociones de espacio y tiempo) y control de la respiración.

\footnotetext{
${ }^{2}$ Así describe Schmidt el campo científico del control motor

${ }^{3}$ Estuvo en Colombia presentando sus trabajos y es autor de un texto clásico en el área.

${ }^{4}$ Como la propuesta de Rossel, pero que destaca la relación entre movimiento y desarrollo mental.

${ }^{5}$ Pick y Vayer proponen la educación de las conductas motrices y ponen en circulación los temas relacionados con el esquema corporal, conductas motrices de base y demás. Luego, estos conceptos tipifican la orientación psicomotriz de la EF durante los años 80.

${ }^{6}$ Aunque esto sucede en Francia en 1971, en español solo se conoce en los 80, con la tercera edición de su libro en 1989

${ }^{7}$ Ver la propuesta de Rigal al respecto, editada en francés en 1985 y traducida por Pila Teleña en 1987.
} 
La fisiología del ejercicio es un filón de conocimientos que afectó la EF trascendentalmente. Aunque el tema de la salud es también un objetivo de la EF, fue el trabajo físico el que movió a Astrand y Rhodal en Suecia a investigar. Desde finales de los 70, el profesor Astrand ha liderado este campo, y sus propuestas no solo permearon la $E F$, sino que transformaron la medicina. Los trabajos en éste sector han sido continuados por muchos académicos, como Fox y Mathews o Costill (1984). Se ha establecido, además un proceso de diferenciación temática y ha surgido la fisiología del esfuerzo, la de los deportes, la de los jóvenes (Bar-Or y Rowland, p. ej.), la de altura, la de las profundidades, etc. Se reconocen campos afines como el de la nutrición para el esfuerzo o el deporte, y el de la hidratación en los esfuerzos físicos. El enfoque metodológico clásico de estos campos ha sido el experimental. En Alemania el tema se desarrolla igualmente, y se estudian a fondo las bases biológicas del ejercicio y del entrenamiento. ${ }^{8}$

En el Departamento de EF, el impacto de la temática es grande. Se instala la asignatura como obligatoria luego de pasar como opcional, proceso semejante al de la cátedra de psicomotricidad. Se desarrollan algunas actividades de laboratorio y se le otorga un reconocimiento especial dentro de la formación de los profesores de Educación Física.

Los años 80 se presentaron como una época de discusión sobre la fundamentación de la teoría de la educación física. Cajigal, en España, recogió ideas alemanas y propuso una EF humanística, analizó la estructura y funcionamiento del deporte con su metáfora del árbol ${ }^{9}$ y reflexionó sobre el movimiento humano como objeto de estudio de la EF Se tradujo al español en 1976 el trabajo de Ommo Gruppe (planteado en 1965 en alemán), que refleja una propuesta crítica de teoría pedagógica en la EF. Tal vez, estos trabajos junto con los de los soviéticos sobre la cultura física ${ }^{10}$, permitieron visualizar que en Europa existía una discusión sobre la problemática teórica de la EF; se podría decir que era un intento de fundamentación serio de la disciplina. Una propuesta resulta interesante y fundamentada en esta etapa: la de Kurt Meinel. Parece tratarse de una didáctica del movimiento, pero en realidad es toda una teoría del movimiento para los ámbitos de la EF y del deporte, con fundamentación ideológica ${ }^{11}$. Repercusiones de estas reflexiones se verán posteriormente en países diversos. Cuba, por ejemplo, presenta en los últimos años de los 80 su postura al respecto ${ }^{12}$.

El impacto sobre la orientación del departamento de EF. es interesante. El carácter humanístico de Cajigal impresiona. Se le conoce personalmente en sus visitas y cursos en Bogotá, organizados por el profesor Héctor Peralta y el Comité Nacional de la Federación Internacional de Educación Física (FIEP). Las propuestas de Cajigal se acogen y se critican las anteriores, por su falta de pedagogía humanista.

Uno de los temas de los 90 sin duda fue el del entrenamiento deportivo. La teoría del entrenamiento venía desarrollándose en la práctica, hasta que Matveev sistematiza sus experiencias con los equipos nacionales soviéticos, desde la Escuela de Deportes de la Unión Soviética. Sus observaciones, experiencias directas, análisis teóricos y generalizaciones desde la matemática, contribuyeron a ordenar y revolucionar los

\footnotetext{
${ }^{8}$ Como el trabajo de Nocker de 1980 sobre el tema, editado en alemán con anterioridad.

${ }^{9}$ Ver su obra ;Oh, deporte!

${ }^{10}$ Lev Matveev escribe en 1979 sobre la información de la Teoría General de la Cultura Física y el Depoprte. Ver su articulo en español en Ciencias Sociales Contemporáneas. Ed. Nueva colombia.

${ }^{11}$ La propuesta es desarrollada en la Escuela Superior Alemana de Cultura física en Leipzing, presentada en alemán en 1971 y en español en 1977. Su fuente política es el socialismo.

${ }^{12}$ Caridad calderón sintetiza algunos aspectos sobre la teoría de la EF como disciplina científica y de la instrucción, que se puede considerar influenciada por EF soviética y alemana oriental.
} 
sistemas de entrenamiento deportivo de gran parte del mundo. Su trabajo principal es conocido en español en 1977, nuevamente por el esfuerzo del INEF de Madrid, quien lo traduce del alemán. Los deportes referenciados son la natación, la halterofilia y el atletismo, por su facilidad para manejar resultados analizables en deporte adulto y de alto rendimiento. Hace toda una discusión sobre el tema, sobre su evolución y recoge sus experiencias en la propuesta de periodización del entrenamiento deportivo.

Los estudios y propuestas de los años 90 en la teoría de la EF pueden recogerse en la reflexión sobre la formación corporal y el concepto del cuerpo en las diferentes concepciones de EF. Los planteamientos filosóficos, sociológicos y psicológicos sobre el cuerpo son conocidos por parte de profesores del Departamento, y acogidos por algunos, sin reserva, como nuevo centro de la EF. Este conocimiento sobre el tema del cuerpo, le propone a la EF un nuevo núcleo de problemas, que la psicología, la antropología, la sociología y la filosofía habían abordado, pero no la EF.

De ésta manera, la teoría de la educación física se acerca al tema de la corporeidad y de las experiencias corporales, como temas que sirvieron para un nuevo enfoque pedagógico.

Hacia finales de los años 90, se presenta(n) la (s) ciencia (s) del deporte. Es un intento de origen alemán (!) de recoger los trabajos que diferentes disciplinas han hecho sobre el deporte, y diferentes teóricos han intentado fundamentar. El tema no es nuevo, pues en 1939 Altrock escribió un articulo sobre "Tareas y extensión de la ciencia del deporte». Más recientemente, Gruppe contribuyó con más artículos al respecto, enfatizando el desarrollo de ésta disciplina joven. Pero ha sido Haag ${ }^{13}$ quien desde 1979 ha impulsado la idea de establecer el campo con un modelo de siete disciplinas: medicina del deporte, biomecánica deportiva, psicología deportiva, pedagogía deportiva, sociología deportiva, historia deportiva y filosofía deportiva. Cada una de éstas disciplinas tiene su origen en una de referencia. Adicionalmente, Barreau y Morne (1984) plantean que desde mediados de los 70 , se han presentado estudios y obras sobre las actividades corporales y el deporte, que sin duda son del campo de las disciplinas antropológicas. Luschen y Weis en 1976 plantearon su sociología del deporte (en español en 1979), desarrollando teorías que explican los comportamientos, la estructura y el funcionamiento social del deporte en diferentes aspectos. En 1990, en España se estudian los aspectos sociales del deporte desde una mirada sociológica. La biomecánica deportiva es disciplina científica desde 1962 en Leipzig y está en un alto nivel de desarrollo, incluso con procesos estandarizados de pruebas en deportes. El trabajo de metrología deportiva de Zatziorsky es un modelo de estudio, lamentablemente conocido tardíamente en español.

Estos temas influyeron en la orientación de los profesionales en EF, que pudieron asistir a la especialización en pedagogía del entrenamiento deportivo que la Facultad logró brindar a la comunidad. Sin embargo su incidencia apenas comienza. No hay que olvidar que Cuba, desde el mismo enfoque, tiene aportes significativos sobre algunos deportes.

\section{Reflexiones}

Al parecer la teoría de la educación física se ha nutrido de distintas tendencias temáticas en los diferentes momentos de la Facultad. La acogida de los conceptos básicos de cada tendencia ha sido permanente. Es posible que se trate del proceso de construcción de un objeto amplio de estudio de la EF Debe destacarse que en su momento, la nueva temática acogida se interpreto como orientación determinante de la formación profesional. Esto generó dinámica de discusión teórica, caracterizada por una

\footnotetext{
${ }^{13}$ Ver la elaboración que el Iternational Council of Sport and Physical Educatión presentó en una obra de Haag, denominada Fundamentación teórica de la ciencia del deporte como disciplina científica.
} 
crítica sobre el anterior énfasis, a veces racionalizada, pero probablemente emotiva y caracterizada por una «santificación» de lo nuevo.

Se debe destacar la labor del INEF de Madrid en la difusión de conocimientos de EF, traduciendo en los 70 de diferentes idiomas al español. En años recientes, España continúa trabajando en la senda delineada por el INEF, pero también hay que reconocer que a través de Cuba se ha llegado a difundir mucha literatura alemana y soviética de gran impacto científico. Hoy en día, la transferencia de conocimientos en EF es de muy alto nivel. Existen journals o revistas periódicas en todas las disciplinas mencionadas, innumerables obras de texto y de difusión de trabajos científicos y redes de información (como la Atlantis, que es la mayor base de datos del mundo en español con más de 6.400 referencias, o la Red sport.com). La Facultad debe pensar su postura en estos tiempos de gran circulación de información. La acogida de temas puede no ser el paradigma de construcción teórica de la EF, como tampoco la renuncia a lo anterior, por lo novedoso. ¿Cuál debe ser el balance entre tradición y cambio? Es posible que solo la investigación nos permita resolver la cuestión, pero parece que hemos entendido la investigación de diversas maneras y sin acuerdo social ni teórico. Algunos la piensan como la formación de la actitud investigativa y otros como estudio y resolución de preguntas en forma grupal. Este ítem debe ser pensado y analizado.

En el tema de la pedagogía de la EF, los trabajos de Muska Mosston fueron reveladores y de impacto hace algunos años; el de Arnold sobre EF, movimiento) y currículo más actual, permite apreciar otras dimensiones sobre el valor del deporte y del movimiento en la escolarización. La tendencia pedagógica ha saltado de orientación en orientación en la Facultad y últimamente de una consideración de lo social, como la clave de lo pedagógico, se saltó a la experiencia corporal como paradigma. Este hecho debe analizarse para los nuevos proyectos curriculares de EF.

La estadística como herramienta de trabajo científico es común en la EF internacional. El uso de tests, y de evaluaciones con pruebas, han destacado la importancia de ésta disciplina. Los trabajos de Zatziorsky en su metrología deportiva en la Unión Soviética, o de Saftit y Thomas y Nelson en Estados Unidos son ejemplares. En Colombia solo el trabajo de Jáuregui merece destacarse en esta área. Sin embargo, nuestra Facultad no ha dimensionado su uso en la formación profesional y como base de algunas tendencias investigativas.

Se puede reconocer, en el ámbito internacional, el nivel de desarrollo de los temas destacados, como puede verificarse por estudios e investigaciones en la literatura (libros o revistas, o redes), su problematización y estudio en seminarios y congresos o eventos académicos similares, como temáticas o asignaturas en los planes de estudio) de universidades de distintos países, ó asumidos como línea de estudio en laboratorios y centros de investigación en diversas partes del mundo. En Colombia y en la UPN no podemos desconocer estos contenidos que tan arduamente se han construido a lo largo de la historia de la EF. Así, el último tema que no podemos despreciar es el de la historia de la EF. 